\title{
Cerebral Perfusion and Sensory Testing Results Differ in Interstitial Cystitis/Bladder Pain Syndrome Patients with and without Fibromyalgia: A Site-Specific MAPP Network Study
}

\author{
Georg Deutsch' \\ Hrishikesh Deshpande' \\ $\mathrm{H}$ Henry Lai ${ }^{2}$ \\ Jason J Kutch ${ }^{3}$ \\ Timothy J Ness ${ }^{4}$ \\ 'Department of Radiology, University of \\ Alabama at Birmingham, Birmingham, AL, \\ USA; ${ }^{2}$ Division of Urologic Surgery, \\ Department of Surgery, Washington \\ University School of Medicine, St. Louis, \\ MO, USA; ${ }^{3}$ Division of Biokinesiology and \\ Physical Therapy, University of Southern \\ California, Los Angeles, CA, USA; \\ ${ }^{4}$ Department of Anesthesiology and \\ Perioperative Medicine, University of \\ Alabama at Birmingham, Birmingham, \\ AL, USA
}

Correspondence: Timothy | Ness

Department of Anesthesiology, University of Alabama at Birmingham, BMR2-208;

90I 19th St. S, Birmingham, AL, 35205, USA

Tel + I 205-975-9643

$\mathrm{Fax}+\mathrm{I}$ 205-934-7437

Email tness@uabmc.edu
Purpose: Fibromyalgia is a common co-morbidity in patients with interstitial cystitis/bladder pain syndrome. Quantitative sensory testing measures and regional cerebral blood flow measures have been noted to differ from healthy controls in both subjects with fibromyalgia and those with interstitial cystitis when studied independently. The present study examined such measures in subjects with the diagnosis of interstitial cystitis both with and without the co-diagnosis of fibromyalgia to determine whether differences in these measures may be associated with comorbidity.

Patients and Methods: Female subjects with the diagnosis of interstitial cystitis with $(\mathrm{n}=15)$ and without $(n=19)$ the co-diagnosis of fibromyalgia as well as healthy control subjects $(n=41)$ underwent quantitative sensory testing. A subset of these patients ( 9 with and 9 without fibromyalgia) underwent brain perfusion studies using arterial spin labeled functional magnetic resonance imaging. An analysis was performed of absolute regional cerebral blood flow of regions-of-interest when experiencing a full bladder compared with an empty bladder.

Results: Subjects with both interstitial cystitis and fibromyalgia were more hypersensitive than those without fibromyalgia as well as healthy controls in most sensory measures except heat. Subjects with interstitial cystitis, but no fibromyalgia, differed from healthy controls only in toleration of the ischemic forearm task. Other co-morbidities were more common in those subjects with both interstitial cystitis and fibromyalgia. Bladder fullness was associated with significantly greater whole brain gray matter blood flow in subjects with interstitial cystitis and fibromyalgia when compared with that of subjects with interstitial cystitis without fibromyalgia. Examination of regional cerebral blood flow in individual regions-of-interest demonstrated statistically significant differences between the subjects with interstitial cystitis with and those without fibromyalgia bilaterally in the thalamus, amygdala and hippocampus, as well as the right prefrontal cortex and greater responsiveness to changes in bladder fullness in the insula.

Conclusion: Quantitative sensory testing and brain perfusion data support that there are two phenotypes of interstitial cystitis patients, which can be differentiated by a co-diagnosis of fibromyalgia. This may affect responsiveness to treatment and suggest the utility of stratifying interstitial cystitis patients according to their co-morbidities.

Keywords: arterial spin labelling, fMRI, interstitial cystitis, QST

\section{Introduction}

Bladder hypersensitivity is a hallmark feature of the disorder interstitial cystitis/bladder pain syndrome (IC/BPS). Additional modalities of deep tissue hypersensitivity have 
also been noted in subjects with IC/BPS (ICs), which include hypersensitivity in quantitative sensory testing (QST) to muscular and abdominal probing and reduced tolerance to forearm ischemia. ${ }^{1-3}$ IC/BPS has also been noted to have multiple co-morbidities, which include disorders such as irritable bowel syndrome, chronic fatigue syndrome, vulvodynia and fibromyalgia (FM). In particular, FM has also been noted to be associated with deep tissue hypersensitivity on QST. ${ }^{4}$ Previous reports from the Multidisciplinary Approach to the Study of Chronic Pelvic Pain (MAPP) Research Network have observed that ICs with the hallmark feature of FM, diffuse widespread pain, appear to differ in multiple neurophysiological measures from ICs not reporting similar widespread pain. ${ }^{5,6}$ This suggests that there may exist different populations of ICs with potentially different mechanisms related to their pain, thereby requiring different types of treatments. Previously published QST studies have always had limited samples unable to address this important topic. Therefore, we pooled together QST data from our MAPP site-specific study with QST data from other funded studies performed at the University of Alabama at Birmingham ${ }^{1,7,8}$ to give sufficient statistical power to determine if there was evidence for subpopulations of ICs in our overall IC/BPS patient population. We previously identified that ICs, when compared with healthy control subjects, had distinctly different alterations in regional cerebral blood flow (rCBF) measures when experiencing a full bladder. ${ }^{9}$ Since completion of this study, we have expanded our patient sample such that a comparison of rCBF was possible between ICs with a codiagnosis of FM (IC-FMs) and ICs without a co-diagnosis of FM (IC-noFMs) in order to determine whether any observed QST differences were reflected in $\mathrm{rCBF}$ measures.

\section{Patients and Methods Study Summary}

These studies were approved by the University of Alabama at Birmingham's Institutional Review Board for Human Studies (UAB IRB protocol numbers F060505003, F091105005, F080417007) and were conducted in accordance with the Declaration of Helsinki. All ICs were recruited from clinical populations treated by one of the investigators (TJN); all HCs were recruited by advertisements in institutional publications, which prompted them to call a contact phone number for the study coordinator who then described the study. All subjects had the procedures to be performed extensively explained to them and given informed consent prior to participation in the study.
All subjects were given monetary compensation for their participation. In total, 75 female subjects (34 subjects meeting criteria for IC/BPS ${ }^{10,11}$ and $41 \mathrm{HCs}$ ) underwent QST as described below in conjunction with either the MAPP Research Network ${ }^{12,13}$ (protocol M089) as a sitespecific study or as part of the other QST studies performed locally. ${ }^{1,7-9}$ The MAPP Research Network studies were the last of these studies performed, and as part of that protocol, all IC subjects studied (9 IC-FMs; 9 IC-noFMs) had $\mathrm{rCBF}$ measures performed in addition to QST measures. fMRI data related to HCs have been previously reported $^{9}$ and so are not repeated here since the focus of the present study was on differences between subsets of IC subjects. All subjects also filled out multiple questionnaires and reported other health history including any diagnoses of painful conditions. FM, irritable bowel syndrome, chronic fatigue syndrome or vulvodynia diagnoses were assigned using standardized criteria. ${ }^{12,13}$ All ICs met current American Urological Association criteria for IC/ BPS $^{10}$ and in addition had undergone cystoscopic evaluation, which demonstrated the presence of glomerulations. Other confusable diagnoses had also been assessed and so these subjects also met the more stringent diagnostic criteria for interstitial cystitis, which had previously been established by the NIDDK ${ }^{11}$ in the 1990s.

\section{Summary of QST Methods}

All QST measures were performed by one of the investigators (TJN) or individuals and actively supervised and trained by that investigator. Methods are the same as described in previous publications ${ }^{1,8}$ and consisted of hot pain tolerance measured in ${ }^{\circ} \mathrm{C}$ obtained using a Medoc TSA-2001 unit applied to the forearm, ice water hand immersion time tolerances in sec (maximum $120 \mathrm{~s}$ ), muscular pressure thresholds measured in $\mathrm{mP}$ using a handheld algometer applied to the trapezius midpoint between the neck and shoulder and a tourniquet and exercise-linked ischemic forearm tolerance task measured in seconds tolerated (tourniquet released on request).

\section{Summary of ASL-fMRI Methods}

All subjects had a high-resolution MRI image and ASLfMRI measures performed in the "Full Bladder" condition (patients self-rated that they were near their maximally tolerated bladder capacity) and in the "Empty Bladder" (post void) condition using methodology and analysis as described in a previous publication; ${ }^{9} 11$ of the 18 ICs reported here had data included in that previous report. 
Briefly, subjects were given oral hydration and encouraged to consume as much as possible while doing the paperwork associated with the study. ASL-fMRI scans were timed to either immediately follow complete voiding of the bladder (the Empty Bladder condition) or when subjects reported a strong urge to void (Full Bladder condition) with the order of the two scans randomized according to the patients' preferences. Scans were obtained using a 3T MR Philips Achieva MRI scanner. Motion-corrected ASL scans were compartmentalized into groups of control or labeled images. Quantitative CBF maps were calculated from the control-tag perfusion signal difference using a modified two-compartment ASL perfusion model: ${ }^{13}$

$$
f=\Delta M l R_{1 a} \exp \left(w R_{1 a}\right) / 2 M_{o} a x\left[1-\exp \left(-t R_{1 a}\right)\right]^{-1}
$$

where $f$ is $\mathrm{CBF}, \Delta M$ is the difference signal between the control and label acquisitions, $R_{l a}$ is the longitudinal relaxation rate of blood, $\tau$ is the labeling time, $\omega$ is the post-labeling delay time, $\alpha$ is the labeling efficiency, $\lambda$ is blood/tissue water partition coefficient, and $M_{o}$ is approximated by the control image intensity. The parameters used in this study were $R_{1 a}=1 / 1664 \mathrm{sec}, \alpha=0.85, \lambda=0.09 \mathrm{~g} / \mathrm{mL}$, $\tau=2.4 \mathrm{sec}, \omega=1.4 \mathrm{sec}$. $\mathrm{rCBF}$ maps so generated were normalized to the Montreal Neurology Institute template and smoothed using a 8x8x8 mm Gaussian kernel. Based on the existing literature on pain imaging as referenced in our previous report ${ }^{9}$ we selected bilateral cortical and subcortical regions of interest (ROIs) as well as global hemispheric measures bilaterally and an ROI centered on the periaqueductal gray matter. These ROIs were selected with the Wake Forest University pick atlas, which uses the Talairach Daemon database. ${ }^{14}$

\section{Summary of Questionnaire Information}

All the subjects reported a $0-10$ verbal numerical report (0-none, 10-worst ever) rating of pain with voiding which was elicited on the day of QST. ICs that underwent $\mathrm{rCBF}$ measures also filled out Polysyndromic Polysymptomatic Questionnaires, ${ }^{15}$ which quantify ongoing patient symptomatology, as well as multiple forms for the MAPP Network, ${ }^{12,13}$ which included the Brief Pain Inventory and the Complex Medical Symptom Inventory.

\section{Statistical Analysis}

Presented data represent means \pm SEM unless otherwise stated. Continuous demographic and QST data were compared using $t$-tests with Bonferroni correction for multiple measures where appropriate. A power analysis using a difference of 2 in the 11 point "Pain with Voiding" score and an overall standard deviation of 1.9 derived from our previous report of QST measures ${ }^{8}$ indicated a sample size of $15 \mathrm{ICs}$ per group was needed in order to observe an $80 \%$ chance of observing such a difference with a $\mathrm{p}<0.05$. Available data consisted of 34 ICs and so data from all subjects was included in QST measures. The rCBF studies were exploratory in nature and so no power analysis was applied. Categorical data were compared using Fisher's (chi-square) test. Absolute rCBF values $(\mathrm{mL} / 100 \mathrm{~g} / \mathrm{min})$ were utilized in the analysis and in data tables. For rCBF comparisons, a repeated measures ANOVA was performed using two Groups (IC-FM and ICnoFM) with two Conditions (Empty Bladder and Full Bladder) analysis in the selected ROIs. Post hoc comparisons were performed using unpaired $t$-tests.

\section{Results}

\section{Demographics and Physiological Measures}

Overall ICs were older than HCs and had a significantly higher heart rate and blood pressure when assessed in the larger sample (see Table 1). Painful co-morbidities in the ICs, as identified by the methods of the MAPP study, included FM, irritable bowel syndrome, chronic fatigue syndrome and vulvodynia. By definition, the HCs did not have these co-morbidities. IC-FMs had statistically more of these co-morbidities than IC-noFMs in both the larger sample (Table 1) and in the subgroup studied using fMRI (Table 2). Notably, use of daily narcotics was similar in these two IC groups (Table 2) and so unlikely an etiology of differences in measures. Headaches (migraines and nonmigraines not differentiated) were common in all groups (HCs and IC subgroups) and so are not examined further.

\section{QST Measures}

Similar to previous reports, ${ }^{1,7,8}$ ICs (total sample) had more pain with voiding and less tolerance of pain in the ischemic forearm task measure than $\mathrm{HC}$ subjects. However, when the ICs are stratified into the IC-FM and IC-noFM subgroups, differences between these subgroups become statistically apparent. IC-FM subjects, not surprisingly, have lower thresholds for eliciting pain from muscle pressure, but they also have greater reports of pain with voiding, lower ice water immersion tolerance and lower ischemic forearm task tolerance than IC-noFMs. In all of these measures, the IC-FMs also differ from $\mathrm{HC}$ subjects. Notably, heat pain tolerance measures did not differ 
Table I Demographic and Psychophysical Measures in Broad Sample

\begin{tabular}{|c|c|c|c|c|}
\hline Controls & IC Patients-All & Healthy & IC-noFM & IC-FM \\
\hline $\mathrm{N}$ & 34 & 41 & 19 & 15 \\
\hline Age & $43 \pm 2 * *$ & $36 \pm 2$ & $43 \pm 3 *$ & $42 \pm 3 *$ \\
\hline MAP $(\mathrm{mm} \mathrm{Hg})$ & $91.3 \pm 2.4 *$ & $85.1 \pm 1.6$ & $89.4 \pm 3.6$ & $93.8 \pm 3.0 *$ \\
\hline HR (bpm) & $89.9 \pm 1.9 * *$ & $74.6 \pm 1.7$ & $92.2 \pm 2.3 * *$ & $87.2+3.0 * *$ \\
\hline$\%$ with Headaches & $62 \% * *$ & $27 \%$ & $53 \%$ & $73 \% * *$ \\
\hline$\%$ with IBS & $41 \% * *$ & $0 \%$ (see note) & $11 \% * \#$ & $80 \% * * \#$ \\
\hline$\%$ with CFS & $37 \% * *$ & $0 \%$ (see note) & $16 \% * \#$ & $67 \% * * \#$ \\
\hline$\%$ with Vulvodynia & $37 \% * *$ & $0 \%$ (see note) & $26 \% * \#$ & $73 \% * * \#$ \\
\hline$>2$ Co-morbidities & $50 \% * *$ & $0 \%$ (see note) & $16 \% * \#$ & $93 \% * * \#$ \\
\hline$>3$ Co-morbidities & $41 \% * *$ & $0 \%$ (see note) & $5 \%$ & $73 \% * * \#$ \\
\hline$\%$ on Narcotics & $69 \% * *$ & $0 \%$ (see note) & $68 \% * *$ & $73 \% * *$ \\
\hline Pain with Void $(0-10)$ & $4.2 \pm 0.5 * *$ & $0 \pm 0$ & $3.0 \pm 0.6 * * \#$ & $5.8 \pm 0.6 * * \#$ \\
\hline Trapezius Pressure (mPs) & $2.67 \pm 0.18$ & $3.01 \pm 0.14$ & $3.21 \pm 0.22^{\# \#}$ & $1.99+0.17 * * \#$ \\
\hline Heat Pain Tolerance $\left({ }^{\circ} \mathrm{C}\right)$ & $44.7 \pm 0.5$ & $46.1 \pm 0.4$ & $44.7 \pm 0.6$ & $44.6 \pm 0.8$ \\
\hline Ice Water Tolerance (s) & $36.4 \pm 4.5$ & $38.8 \pm 3.8$ & $47.7 \pm 6.4^{\# \#}$ & $21.0 \pm 3.2^{* * \#}$ \\
\hline Ischemic Pain Tolerance (s) & $|80 \pm 2|^{* *}$ & $422 \pm 40$ & $216 \pm 33^{* \#}$ & $130 \pm 19 * * \#$ \\
\hline
\end{tabular}

Notes: See text for description of diagnoses and measures. Statistical tests consisted of unpaired $t$-tests or Chi squared tests as appropriate. $* * * *^{*}<0.05, \mathrm{p}<0.0 \mathrm{I}-\mathrm{different}$ from Healthy Controls. $\#, \#<0.05, P<0.01$ - for IC subgroups - different from other subgroup. Patients were excluded from the Healthy Control group if they had a diagnosis of any chronic pain disorder except headaches. The IC Patients-All data is a combination of the IC-noFM and IC-FM data $>2$ and $>3$ Co-morbidities include IC, IBS, CFS and vulvodynia but not headache.

Abbreviations: MAP, mean arterial pressure; HR, heart rate; IC, interstitial cystitis; IBS, irritable bowel syndrome; CFS, chronic fatigue syndrome.

between any groups consistent with our previous reports ${ }^{1}$ which only observed hypersensitivity in deep tissue QST measures in subjects with IC/BPS. IC-noFMs only differed statistically from $\mathrm{HCs}$ in their voiding intensities and ischemic forearm task measures. These findings are all summarized in Table 1. The subset of ICs who underwent fMRI measures also received QST measures and statistically significant differences in the same measures as those noted in the larger sample continued to be present in this subset (Table 2). As participants in the MAPP studies, this group also received multiple extra questionnaires and it is notable that the IC-FM group had a statistically higher score using the Polysymptomatic Polysyndromic Questionnaire than the IC-noFM group. The FM-IC group also reported many sites of pain in the Brief Pain Inventory and many symptoms in the Complex Medical Symptom Inventory, although greater, these measures did not statistically differ from the measures obtained from the IC-noFM group (Table 2).

\section{rCBF Analysis}

An initial repeated measures ANOVA was performed on whole brain gray matter rCBF measures, which demonstrated a significant effect of the group assignment (IC$\mathrm{FM}$ versus $\mathrm{IC}$-noFM $)\left[\mathrm{F}_{1,16}=5.057 ; \mathrm{p}=0.039\right]$, a significant effect of condition (Full versus Empty Bladder)
$\left[\mathrm{F}_{1,16}=12.173 ; \mathrm{p}=0.003\right]$ and a group $\times$ condition interaction $\left[F_{1,16}=6.863 ; p=0.019\right]$. These values indicate greater responsiveness of the Whole Brain gray matter to bladder filling in IC-FM subjects when compared with the IC-noFM subjects, although there was no difference in baseline

Table 2 IC Subgroups Undergoing fMRI Measures

\begin{tabular}{|l|l|l|}
\hline & IC-no FM & IC-FM \\
\hline N & 9 & 9 \\
Age & $46.8 \pm 4.9$ & $40.2 \pm 3.3$ \\
BPI Number of Sites & $6.3 \pm 1.4$ & $13.3 \pm 3.4$ \\
PSPSQ Score & $15.3 \pm 3 . I^{*}$ & $25.0 \pm 3.0 *$ \\
CMSI Number of Symptoms & $11.9 \pm 2.1$ & $17.7 \pm 3.1$ \\
\% with IBS & $0 \% * *$ & $89 \% * *$ \\
$\%$ with CFS & $22 \%$ & $56 \%$ \\
$\%$ with Vulvodynia & $44 \%$ & $78 \%$ \\
\% with >2 Co-Morbidities & $22 \% *$ & $100 \% *$ \\
\% with >3 Co-Morbidities & $0 \% * *$ & $78 \% * *$ \\
\% on Narcotics & $78 \%$ & $78 \%$ \\
Pain with Void (0-10) & $2.8 \pm 0.7^{* *}$ & $5.9 \pm 0.8^{* *}$ \\
Trapezius Pressure (mPs) & $2.62 \pm 0.15^{* *}$ & $1.78 \pm 0.13^{* *}$ \\
Ice Water Tolerance (s) & $54 \pm 10^{*}$ & $23 \pm 5^{*}$ \\
Ischemic Pain Tolerance (s) & $303 \pm 49^{* *}$ & $136 \pm 26^{* *}$ \\
\hline
\end{tabular}

Notes: Data represent incidence or Mean \pm SEM. See text for description of diagnoses and measures. Statistical tests consisted of unpaired $t$-tests or Chi squared tests as appropriate. *** $\mathrm{p}<0.05, \mathrm{p}<0.01$ indicates different from other subgroup $>2$ and $>3$ Co-morbidities include that IC, IBS, CFS and vulvodynia.

Abbreviations: IC, interstitial cystitis; IBS, irritable bowel syndrome; CFS, chronic fatigue syndrome; BPI, Brief Pain Inventory; PSPSQ, PolySyndromic PolySymptomatic Questionnaire; CMSI, Complex Medical Symptom Inventory. 
(Empty Bladder condition) rCBFs between the two groups or between the Full Bladder values in the IC-noFM group and the Empty Bladder values in the IC-FM group. Based on the significant ANOVA results for the whole brain, we conducted similar repeated measures ANOVAs of rCBFs in ROIs, which have previously demonstrated by us ${ }^{9}$ to differ from the rCBFs noted in healthy control subjects in order to localize sites of the overall effects that may be uniquely associated with the co-morbidity of FM. Sites with statistically significant effects on the group assignment or with a group $\times$ condition interaction are listed in Table 3 . All the sites examined are reported in Table 4. Notably, in the Empty Bladder condition, there were no statistically significant differences between IC-FMs and IC-noFMs in rCBFs in any of the selected ROIs. In contrast, the right and left amygdala, right and left hippocampus, right and left insula and right and left thalamus ROIs as well as the left prefrontal cortex ROI demonstrated greater rCBF in ICFMs in the Full Bladder condition when compared to the IC-noFMs. In this selected sample of ROIs as well as the larger overall sample, it is apparent that the IC-FM subjects have greater reactivity to bladder filling with Full Bladder rCBFs statistically greater than the Empty Bladder rCBFs in virtually all ROIs examined. In contrast, there were no statistically significant differences in the Full and Empty Bladder mean measures from the IC-noFM subjects in any of the ROIs examined.

\section{Discussion}

The most important finding of the present study is that it gives strong objective evidence for what has long been suspected based on subjective reports: that there are distinct

Table 3 Regional Cerebral Blood Flow (rCBF) Distribution During Empty and Full Bladder States in Selected Regions of Interest Associated with Pain Sensation (Sites with Statistically Significant "Group" or "Group × Condition" Effects)

\begin{tabular}{|c|c|c|c|c|c|}
\hline \multirow{2}{*}{$\begin{array}{l}\text { Region of } \\
\text { Interest (ROI) }\end{array}$} & \multicolumn{2}{|c|}{ IC-noFM Subjects } & \multicolumn{2}{|c|}{ IC-FM Subjects } & \multirow{2}{*}{$\begin{array}{l}\text { Comparative Repeated Measure } \\
\text { ANOVA Statistics }\end{array}$} \\
\hline & Empty & Full & Empty & Full & \\
\hline Whole Brain GM & $54.9 \pm 2.8$ & $56.8 \pm 2.8$ & $57.4 \pm 2.1$ & $70.7 \pm 4.0 *$ & $\begin{array}{l}\text { Group } p=0.039 \text {; Condition } p=0.003 \text {; Group } \times \text { Condition } \\
\text { interaction } p=0.019\end{array}$ \\
\hline rHemisphere GM & $53.7 \pm 2.8$ & $56.3 \pm 3.0$ & $56.8 \pm 2.3$ & $70.1 \pm 4 . I^{*}$ & $\begin{array}{l}\text { Group } p=0.04 I \text {; Condition } p=0.003 \text {; Group } \times \text { Condition } \\
\text { interaction } p=0.033\end{array}$ \\
\hline IHemisphere GM & $56.0 \pm 3.2$ & $57.3 \pm 2.6$ & $58.1 \pm 2.0$ & $71.3 \pm 4.1^{*}$ & $\begin{array}{l}\text { Group } p=0.041 \text {; Condition } p=0.005 \text {; Group } \times \text { Condition } \\
\text { interaction } p=0.016\end{array}$ \\
\hline rAmygdala & $52.3 \pm 4.0$ & $52.7 \pm 4.1$ & $54.6 \pm 4.4$ & $69.9 \pm 4.0 *$ & $\begin{array}{l}\text { Group } p=0.032 \text {; Condition } p=0.073 ; \text { Group } \times \text { Condition } \\
\text { interaction } p=0.089\end{array}$ \\
\hline IAmygdala & $52.3 \pm 4.5$ & $54.0 \pm 3.4$ & $56.7 \pm 2.1$ & $71.0 \pm 6.1 *$ & $\begin{array}{l}\text { Group } p=0.025 \text {; Condition } p=0.073 \text {; Group } \times \text { Condition } \\
\text { interaction } p=0.156\end{array}$ \\
\hline rHippocampus & $48.4 \pm 4.0$ & $52.3 \pm 3.5$ & $53.6 \pm 3.8$ & $\begin{array}{c}72.5 \\
\pm 4.7 * *\end{array}$ & $\begin{array}{l}\text { Group } p=0.009 \text {; Condition } \mathbf{p}=\mathbf{0 . 0 0 8} \text {; Group } \times \text { Condition } \\
\text { interaction } p=0.064\end{array}$ \\
\hline IHippocampus & $50.4 \pm 3.4$ & $54.4 \pm 2.8$ & $56.3 \pm 2.5$ & $72.8 \pm 6.4^{*}$ & $\begin{array}{l}\text { Group } p=0.008 ; \text { Condition } p=0.024 \text {; Group } \times \text { Condition } \\
\text { interaction } p=0.152\end{array}$ \\
\hline rThalamus & $49.5 \pm 3.6$ & $49.0 \pm 3.4$ & $45.8 \pm 2.3$ & $\begin{array}{c}69.8 \\
\pm 4.6 * *\end{array}$ & $\begin{array}{l}\text { Group } p=0.025 \text {; Condition } p=0.006 \text {; Group } \times \text { Condition } \\
\text { interaction } p=0.004\end{array}$ \\
\hline IThalamus & $47.7 \pm 3.7$ & $50.1 \pm 3.8$ & $46.5 \pm 2.1$ & $\begin{array}{c}69.8 \\
\pm 4.5 * *\end{array}$ & $\begin{array}{l}\text { Group } p=0.006 ; \text { Condition } p=0.008 ; \text { Group } \times \text { Condition } \\
\text { interaction } p=0.025\end{array}$ \\
\hline rinsula & $50.9 \pm 4.2$ & $54.7 \pm 4.4$ & $48.8 \pm 3.3$ & $69.9 \pm 4.8^{*}$ & $\begin{array}{l}\text { Group } p=0.176 ; \text { Condition } p=0.005 ; \text { Group } \times \text { Condition } \\
\text { interaction } p=0.035\end{array}$ \\
\hline Ilnsula & $52.4 \pm 3.1$ & $53.9 \pm 3.0$ & $49.5 \pm 2.8$ & $68.2 \pm 4.8^{*}$ & $\begin{array}{l}\text { Group } p=0.123 \text {; Condition } p=0.0 I I ; \text { Group } \times \text { Condition } \\
\text { interaction } p=0.027\end{array}$ \\
\hline rPreFrontal & $50.5 \pm 3.4$ & $54.9 \pm 3.0$ & $56.7 \pm 2.8$ & $66.8 \pm 3.8 *$ & $\begin{array}{l}\text { Group } p=0.044 ; \text { Condition } p=0.003 ; \text { Group } \times \text { Condition } \\
\text { interaction } p=0.179\end{array}$ \\
\hline IPreFrontal & $53.7 \pm 2.8$ & $55.8 \pm 3.6$ & $56.1 \pm 3.1$ & $65.1 \pm 3.7$ & $\begin{array}{l}\text { Group } p=0.162 \text {; Condition } p=0.035 \text {; Group } \times \text { Condition } \\
\text { interaction } p=0.173\end{array}$ \\
\hline
\end{tabular}

Notes: See text for full description of groups, measures and analysis. Groups are IC-noFM ( $n=9)$ and IC-FM ( $=9$ ); Conditions are Empty and Full. ****ndicate statistically different from Full Condition in IC-noFM Group with $\mathrm{P}<0.05$ and $\mathrm{p}<0.0 \mathrm{I}$ respectively; precise values for these differences which are Bolded are given in the far right column. There were no statistical differences between Groups in the Empty Condition. 
Table 4 Regional Cerebral Blood Flow (rCBF) Distribution During Empty and Full Bladder States in Selected Regions of Interest Associated with Pain Sensation (Sites Without Statistically Significant "Group” or "Group × Condition” Effects)

\begin{tabular}{|c|c|c|c|c|c|}
\hline \multirow[t]{2}{*}{ Regions of Interest (ROI) } & \multicolumn{2}{|c|}{ IC-NoFMs } & \multicolumn{2}{|c|}{ IC-FMs } & \multirow{2}{*}{$\begin{array}{l}\text { Repeated Measures ANOVA Statistics } \\
\text { FM vs NoFM; Full vs Empty; Interaction }\end{array}$} \\
\hline & Empty & Full & Empty & Full & \\
\hline PAG & $48.6+3.9$ & $53.1+2.1$ & $45.1+3.8$ & $66.0+6.7^{*}$ & $p=0.264 ; p=0.017 ; p=0.106$ \\
\hline ICerebellum & $59.1+6.0$ & $63.2+3.7$ & $63.8+4.1$ & $77.2+5.1^{\#}$ & $p=0.157 ; p=0.005 ; p=0.105$ \\
\hline rCerebellum & $58.6+5.8$ & $61.3+3.4$ & $62.9+4.8$ & $77.0+4.7^{\#}$ & $p=0.114 ; p=0.015 ; p=0.086$ \\
\hline IAnt Cingulate & $50.1+4.3$ & $56.6+3.1$ & $55.6+5.2$ & $65.0+2.9$ & $p=0.156 ; p=0.021 ; p=0.641$ \\
\hline rAnt Cingulate & $46.8+3.8$ & $55.1+3.5$ & $49.5+4.7$ & $63.4+2.9^{\#}$ & $p=0.173 ; p=0.008 ; p=0.455$ \\
\hline IMid Cingulate & $41.8+3.6$ & $47.0+4.2$ & $38.2+4.0$ & $54.2+2.6^{\#}$ & $p=0.605 ; p=0.015 ; p=0.183$ \\
\hline rMid Cingulate & $43.4+3.5$ & $49.4+3.6$ & $40.3+3.9$ & $56.5+2.9^{\#}$ & 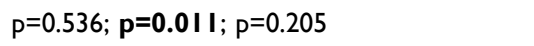 \\
\hline IPost Cingulate & $44.6+5.1$ & $49.4+3.9$ & $45.6+3.8$ & $60.7+3.0^{\#}$ & $p=0.171 ; p=0.016 ; p=0.185$ \\
\hline rPost Cingulate & $46.3+5.2$ & $48.2+3.0$ & $45.3+4.0$ & $61.2+2.5^{\#}$ & $p=0.106 ; p=0.045 ; p=0.107$ \\
\hline$|B A|$ & $42.5+3.6$ & $45.4+3.8$ & $43.4+2.7$ & $54.8+3.9$ & $p=0.187 ; p=0.047 ; p=0.217$ \\
\hline rBAI & $40.3+4.2$ & $43.1+3.8$ & $38.1+2.8$ & $50.3+4.4$ & $p=0.516 ; p=0.074 ; p=0.244$ \\
\hline IBA2 & $44.3+3.5$ & $47.3+3.8$ & $42.3+2.9$ & $54.7+3.5^{\#}$ & $p=0.473 ; p=0.033 ; p=0.170$ \\
\hline rBA2 & $42.9+3.1$ & $46.7+3.8$ & $39.0+3.9$ & $56.3+4.5^{\# \#}$ & 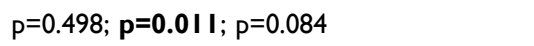 \\
\hline IBA3 & $45.1+3.4$ & $49.2+3.6$ & $43.8+2.7$ & $55.9+3.7$ & $p=0.436 ; p=0.026 ; p=0.252$ \\
\hline rBA3 & $42.1+3.2$ & $47.0+4.4$ & $41.3+3.2$ & $55.7+4.3^{\#}$ & $p=0.325 ; p=0.020 ; p=0.218$ \\
\hline IBA4 & $46.3+3.0$ & $5 I . I+4 . I$ & $44.2+3.2$ & $57.8+4.0$ & $p=0.538 ; p=0.020 ; p=0.238$ \\
\hline rBA4 & $43.6+3.6$ & $46.9+3.4$ & $43.7+3.3$ & $57.0+4.4^{\# \#}$ & $p=0.220 ; p=0.024 ; p=0.151$ \\
\hline IBA6 & $46.7+2.6$ & $50.1+4.1$ & $46.0+2.3$ & $59.5+4.5^{\#}$ & $p=0.271 ; p=0.016 ; p=0.126$ \\
\hline rBA6 & $45.5+3.8$ & $49.6+3.6$ & $44.4+2.2$ & $60.0+4.4^{\# \#}$ & $p=0.259 ; p=0.008 ; p=0.098$ \\
\hline IAngular & $44.3+3.3$ & $46.3+3.4$ & $43.1+3.1$ & $59.6+4.3^{\#}$ & $p=0.090 ; p=0.024 ; p=0.071$ \\
\hline rAngular & $44.1+3.1$ & $46.5+3.2$ & $41.5+3.3$ & $57.9+4.0^{\#}$ & $p=0.217 ; p=0.015 ; p=0.061$ \\
\hline IPreCentral & $45.9+3.0$ & $50.2+3.7$ & $43.4+2.5$ & $59.2+4.0^{\#}$ & $p=0.296 ; p=0.013 ; p=0.128$ \\
\hline rPreCentral & $46.0+4.2$ & $47.8+3.5$ & $43.3+3.0$ & $59.1+4.6^{\#}$ & $p=0.304 ; p=0.029 ; p=0.075$ \\
\hline IPostCentral & $46.8+3.2$ & $50.3+3.7$ & $43.7+2.9$ & $57.1+3.9^{\#}$ & $p=0.588 ; p=0.026 ; p=0.174$ \\
\hline rPostCentral & $42.3+3.5$ & $46.5+3.4$ & $41.0+3.0$ & $56.7+4.3^{\# \#}$ & $p=0.246 ; p=0.012 ; p=0.118$ \\
\hline ISupplemental Motor & $43.1+3.7$ & $48.1+4.8$ & $44.9+3.7$ & $55.7+4.3$ & $p=0.357 ; p=0.022 ; p=0.365$ \\
\hline rSupplemental Motor & $42.6+4.3$ & $50.0+4.4$ & $43.1+2.2$ & $58.3+4.3^{\#}$ & $\mathrm{p}=0.3 \mathrm{II} ; \mathrm{p}=\mathbf{0 . 0 0 7} ; \mathrm{p}=0.296$ \\
\hline
\end{tabular}

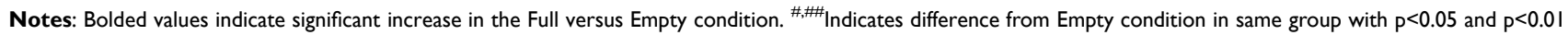
respectively; *Indicates significant difference from same condition in IC-noFM group with $p<0.05$.

subgroups of subjects within the diagnosis of IC/BPS. In this study, IC subjects with and without the diagnosis of FM (based on subjective reports of widespread pain) had objective findings (quantitatively different sensory processing in non-urological sites as well as statistically different regional cerebral blood flow patterns) that correlated with those subjective reports. Notably, this was only one of the many co-morbidities that might have given similar results if examined in a similarly focused fashion and with an adequate statistical sample, but to the best of our knowledge, this is the first objective report of such differences due to co-morbidity in the IC/BPS population. FM was selected as the co-morbidity with the most equal distribution in our patient sample. Additional co-morbidities may prove to be of equal or greater importance as a much greater statistical sample should become available due to the ongoing MAPPII studies, which will have a large sample of phenotyped subjects who will also have been studied with QST and perfusion imaging. ${ }^{16}$ At present, our small sample must be viewed as a potential preview of yet-to-come results. Notably, all the IC subjects included in this study met the more conservative criteria for IC/BPS, ${ }^{10,11}$ which required clinical findings on cystoscopy, which included glomerulations following hydrodistention. It will be important when assessing future studies related to QST and/or cerebral perfusion, determining whether the same diagnostic criteria for IC/BPS are utilized or less stringent symptom-based criteria such as are being used in some ongoing studies. ${ }^{16}$

The presence of multiple co-morbidities was not pathognomonic for the different subgroups, but it is 
notable that having multiple co-morbidities such as irritable bowel syndrome, chronic fatigue syndrome or vulvodynia was the rule in the FM-IC group (93-100\% of the sample), whereas it was the exception in the IC-noFM group (5-16\% of the sample). This was also reflected in the higher Polysymptomatic Polysyndromic Questionnaire scores in IC-FMs compared with IC-noFMs. As a group, it has been reported that ICs have more co-morbidities than other populations, ${ }^{17,18}$ but it is possible that this generalization may be predominantly due to a skew in statistics produced by the polysyndromic phenotype noted in the present study. Krieger et $\mathrm{al}^{19}$ have previously observed that when examined independently, polysyndromic ICs also have more severe urological symptoms, similar to the present study. Others have given evidence for two perceptual phenotypes within other chronic painful disorders, such as irritable bowel syndrome. ${ }^{20}$

Baseline (Empty Bladder condition) rCBF pattern differences between IC-FMs and IC-noFMs were not statistically different and so it is not likely that "empty bladder" fMRI scans will prove to have diagnostic value. In contrast, ASL-fMRI scans obtained during the Full Bladder condition could have diagnostic value, particularly if coupled with imaging of the Empty Bladder condition so that $\mathrm{rCBF}$ reactivity to bladder filling can be assessed. Additional study of patients suffering from other chronic pain disorders including other pelvic pain disorders, such as vulvar vestibulitis and other visceral pain disorders such as irritable bowel syndrome would allow for a determination of the specificity of the measure. These studies allow us to glimpse the differences in central nervous system processing that are present in FM-IC subjects.

The method of ASL-fMRI is non-invasive, temporally brief in acquisition and requires limited instrumentation over standardly available MRI software. This means it could serve as an additional piece of data that informs clinical decisions related to the treatment of IC/BPS. In order to be meaningful in relation to an individual patient, normative values for patient subgroups would need to be established. Our choice to use an ASL-fMRI method was based on a desire to observe absolute differences between IC-FMs and IC-noFMs, as well as patterns of change produced by a natural stimulus. We also desired to utilize methods that would be easy to use clinically. Industry generated ASL software is available for most highresolution MRI units, so it would be possible to translate the findings of the present study to clinical practice with relative ease. Notably, the rCBF responses to bladder filling, which were consistent in the IC-FM subjects, were neither vigorous nor consistent in the IC-noFM group. This suggests that this subset of patients may have additional heterogeneity in etiology and expression of symptoms related to $\mathrm{IC} / \mathrm{BPS}$. It will require a much larger patient sample than the present study in order to tease out any subsets within that group.

Limitations associated with this study include the fact that only female subjects were studied. Whether similar differences in $\mathrm{rCBF}$ are noted in males with the diagnosis of IC/BPS is yet to be determined. This study utilized the more stringent NIDDK consensus panel criteria for $\mathrm{IC}^{11}$ in that all subjects had cystoscopic findings of glomerulations and so it is not known whether these results can be extrapolated to patients assigned to the IC diagnosis using the more symptom-based criteria proposed by the AUA. ${ }^{10}$ One must presume that a population characterized using symptom-based criteria will be even more heterogeneous in nature and may have less symptom chronicity. The precise importance of increased rCBF in specific ROIs is a topic of debate as it may represent functional alterations of CNS processing of sensory inputs or may simply represent the increased importance, or salience, of the particular stimulus to the subject. Given their enhanced symptomatology, one must assume that the sensation of bladder fullness is of especially high importance to IC-FMs and so the results of the present study may simply reflect the differing mental alertness to sensory cues of the IC-FM subjects with that of the IC-noFM subjects. The fact that a "global" increase in perfusion is noted in the IC-FMs suggests that the activation of specific nodes associated with pain may be less important than the overall activation of all nodes during sensory tasks. Coupled with the present study, other converging lines of evidence give strong support for the assertion that CNS function differs between the IC-FM and IC-noFM subjects and supports the existence of distinctly differing phenotypes of IC/BPS.

An issue of continued debate which is related to the observation of two phenotypes within chronically painful disorder populations is whether these phenotypes might not represent a single phenotype but are at different stages of symptom development. Namely, with prolonged pain, it has been proposed that symptoms become more generalized and subjects therefore become polysymptomic with the result being an acquisition of diagnostic criteria for symptom-defined syndromes. Rodriguez et $\mathrm{al}^{21}$ recently addressed this issue in relation to ICs using MAPP 
Network data and reported that urological symptom duration was not associated with pain severity or with codiagnosis of other chronic overlapping disorders. It is notable that when FMs with or without other diagnoses like irritable bowel syndrome, dysmenorrhea or symptomatic diverticulosis are compared, the FM-only subjects report less pain and QST hypersensitivity than the FMs who have other disorders. ${ }^{22}$ Similar findings are present in vulvodynia $^{23}$ and temporomandibular pain disorder ${ }^{24}$ populations when syndrome-only versus polysyndromic subjects are compared. The simplest explanation for all of these observations is that the polysyndromic population is a mechanistically distinct patient population that meets the criteria for multiple symptom-based disorders. Some have proposed this mechanism to be "functional" and considered it synonymous with somatization disorder, ${ }^{25}$ but other etiologies such as developmental insults leading to a globally hypersensitive neurophysiology have not been ruled out. A distinct mechanism suggests that polysymptomatic patients may require analgesic therapeutics that differ from subjects who express only a limited number of symptoms. The positive spin on this conjecture is that an effective therapy for this phenotype, if identified, would be expected to have efficacy in association with subpopulations of multiple disorders. The present set of investigations will stay focused on painful urological disorders and their specific treatment, but there is obvious hope for a more generalized result. At the very least, there is a need to reliably identify specific phenotypes so that appropriate stratification of subjects may be performed prior to experimental therapeutic interventions. A single treatment would not be expected to normalize mechanistically different etiologies.

\section{Conclusions}

In summary, whole brain gray matter as well as specific ROI rCBFs associated with the stimulus of a full bladder differs between IC-FM subjects and IC-noFM subjects. Similarly, QST data and epidemiological data similarly differ. This suggests that pain mechanisms may differ between the two groups and such stratification into patient subgroups may be needed when designing clinical studies assessing therapeutic effectiveness for IC/BPS. Subjects with multiple symptoms meeting multiple syndromic definitions may mechanistically form a common subgroup across multiple disorders such that finding an analgesic treatment useful in that group may prove beneficial to the treatment of subgroups of multiple syndromes.

\section{Abbreviations}

ANOVA, analysis of variance; ASL, arterial spin label; FM, fibromyalgia; HCs, healthy control subjects; IC/BPS, interstitial cystitis/bladder pain syndrome; ICs, IC/BPS patients; IC-noFM, IC/BPS patients without fibromyalgia; IC-FM, IC/BPS patients without fibromyalgia; MAPP, Multidisciplinary Approach to the Study of Chronic Pelvic Pain; fMRI, functional magnetic resonance imaging; QST, quantitative sensory testing; rCBF, regional cerebral blood flow; ROI, regions of interest.

\section{Acknowledgments}

Funding for the Multidisciplinary Approach to the Study of Chronic Pelvic Pain (MAPP) Research Network was obtained under a cooperative agreement from the National Institute of Diabetes and Digestive and Kidney Diseases (NIDDK) and the National Institutes of Health (NIH): U01 DK082315 (Andriole G, Lai H) and DK051413 (Ness T) This article outlines independent research commissioned by the National Institute for Health (NIH). The views expressed in this article are those of the author(s) and are not necessarily those of the NIH, the NIDDK, or the Department of Health.

\section{Disclosure}

Dr H Henry Lai reports grants from NIH, during the conduct of the study; involved in clinical study for Medtronic and Aquinox; consultant for Astella, MicroGenDx, and Biohaven, outside the submitted work. The authors report no other conflicts of interest in this work.

\section{References}

1. Ness TJ, Powell-Boone T, Cannon R, Lloyd LK, Fillingim RB. Psychophysical evidence of hypersensitivity in subjects with interstitial cystitis. J Urol. 2005;173:1983-1987. doi:10.1097/01. ju.0000158452.15915.e2

2. Lai HH, Gardner V, Ness TJ, Gereau RW. Segmental hyperalgesia to mechanical stimulus in interstitial cystitis/bladder pain syndrome: evidence of central sensitization. J Urol. 2014;191:1294-1299. doi:10.1016/j.juro.2013.11.099

3. Fitzgerald MP, Koch D, Senka J. Visceral and cutaneous sensory testing in patients with painful bladder syndrome. Neurourol Urodyn. 2005;24:627-632. doi:10.1002/nau.20178

4. Pavlakovic G, Petzke F. The role of quantitative sensory testing in the evaluation of musculoskeletal pain conditions. Curr Rheumatol Rep. 2010;12:455-461. doi:10.1007/s11926-010-0131-0

5. Kutch JJ, Ichesco E, Hampson JP, et al. Brain signature and functional impact of centralized pain: a multidisciplinary approach to the study of chronic pelvic pain (MAPP) network study. Pain. 2017;158:1979-1991. doi:10.1097/j.pain.0000000000001001 
6. Lai HH, Jemielita T, Sutcliffe S, et al. Characterization of whole body pain in urological chronic pelvic pain syndrome at baseline: a MAPP research network study. J Urol. 2017;198:622-631. doi:10.1016/j. juro.2017.03.132

7. Powell-Boone T, Ness TJ, Cannon R, Lloyd LK, Weigent DA, Fillingim RB. Menstrual cycle affects bladder pain sensation in subjects with interstitial cystitis. $J$ Urol. 2005;174:1832-1836. doi:10.1097/01.ju.0000176747.40242.3d

8. Ness TJ, Lloyd LK, Fillingim RB. An endogenous pain control system is altered in subjects with interstitial cystitis. $J$ Urol. 2014;191:364-370. doi:10.1016/j.juro.2013.08.024

9. Deutsch G, Deshpande H, Frölich MA, Lai HH, Ness TJ. Bladder distension increases blood flow in pain-related brain structures in subjects with interstitial cystitis. $J$ Urol. 2016;96:902-910. doi:10.1016/j.juro.2016.03.135

10. Hanno PM, Burks DA, Clemens JQ, et al. AUA guideline for the diagnosis and treatment of interstitial cystitis/bladder pain syndrome. J Urol. 2011;185:2162-2170. doi:10.1016/j.juro.2011.03.064

11. Pontari MA, Hanno PM, Wein AJ. Logical and systematic approach to the evaluation and management of patients suspected of having interstitial cystitis. Urology. 1997;49:114. doi:10.1016/S00904295(97)00184-2

12. Landis JR, Williams DA, Lucia MS, et al. The MAPP research network: design, patient characterization and operations. BMC Urol. 2014;14:58. doi:10.1186/1471-2490-14-58

13. Clemens JQ, Mullins C, Kusek JW, et al. The MAPP research network: a novel study of urologic chronic pelvic pain syndromes. $B M C$ Urol. 2014;14:57. doi:10.1186/1471-2490-14-57

14. Lancaster JL, Woldorff MG, Parsons LM, et al. Automated Talairach atlas labels for functional brain mapping. Hum Brain Mapp. 2000;10:120-131. doi:10.1002/1097-0193(200007)10:3<120::AIDHBM30>3.0.CO;2-8

15. Lai HH, North CS, Andriole GL, et al. Urological symptoms in a subset of patients with urological chronic pelvic pain syndrome and a polysymptomatic polysyndromic pattern of presentation. J Urol. 2014;191:1802-1807. doi:10.1016/j.juro.2013.12.031

16. Clemens JQ, Mullins C, Ackerman AL, et al. Urologic chronic pelvic pain syndrome: insights from the MAPP research network. Nature Revs Urol. 2019;16:187-200. doi:10.1038/s41585-018-0135-5
17. Aaron LA, Buchwald D. A review of the evidence for overlap among unexplained clinical conditions. Ann Int Med. 2001;134:868-891. doi:10.7326/0003-4819-134-9 Part 2-200105011-00011

18. Bullones Rodgriguez MA, Afari N, Buchwald DS; the NIDDK Working Group on Urological Chronic Pelvic Pain. Evidence for overlap between urological and nonurological unexplained clinical conditions. $J$ Urol. 2013;189:S66-S76. doi:10.1016/j. juro.2012.11.019

19. Kieger JN, Stephens AJ, Landis JR, et al. Relationship between chronic nonurological associated somatic syndromes and symptom severity in urological chronic pelvic pain syndromes: baseline evaluation of the MAPP study. J Urol. 2015;193:1254-1262. doi:10.1016/j.juro.2014.10.086

20. Naliboff BD, Munakata J, Fullerton S, et al. Evidence for two distinct perceptual alterations in irritable bowel syndrome. Gut. 1997;41:505-512. doi:10.1136/gut.41.4.505

21. Rodriguez L, Stephens AJ, Clemens JQ, et al. Symptom duration in patients with urologic chronic pelvic pain syndrome is not associated with pain severity, neurological syndromes and mental health symptoms: a Multidisciplinary Approach to the study of chronic Pelvic Pain Network study. Urology. 2019;124:14-22. doi:10.1016/j. urology.2018.11.015

22. Costantini R, Affaitati G, Wesselmann U, Czakanski P, Giamberardino MA. Visceral pain as a triggering factor for fibromyalgia symptoms in comorbid patients. Pain. 2017;158:1925-1937. doi:10.1097/j.pain.0000000000000992

23. Nguyen RH, Veasley C, Smolenski D. Latent class analysis of comorbidity patterns among women with generalized and localized vulvodynia: preliminary findings. J Pain Res. 2013;6:303-309. doi:10.2147/JPR.S42940

24. Dahan H, Shir Y, Velly A, Allison P. Specific and number of comorbidities are associated with increased levels of temporomandibular pain intensity and duration. $J$ Headache Pain. 2015;16:528. doi:10.1186/s10194-015-0528-2

25. Lai HH, North CS, Andriole GL, Sayuk GS, Hong BA. Polysymptomatic, polysyndromic presentation of patients with urological chronic pelvic pain syndrome. J Urol. 2012;187:2106-2112. doi:10.1016/j.juro.2012.01.081
Journal of Pain Research

\section{Publish your work in this journal}

The Journal of Pain Research is an international, peer reviewed, open access, online journal that welcomes laboratory and clinical findings in the fields of pain research and the prevention and management of pain. Original research, reviews, symposium reports, hypothesis formation and commentaries are all considered for publication. The manuscript management system is completely online and includes a very quick and fair peer-review system, which is all easy to use. Visit http:// www.dovepress.com/testimonials.php to read real quotes from published authors. 